\title{
A Two-step Segmentation Method for Breast Ultrasound Masses Based on Multi-resolution Analysis
}

\author{
Rafael Rodrigues $^{\mathrm{a}}$, Rui Braz ${ }^{\mathrm{b}}$, Manuela Pereira ${ }^{\mathrm{b}}$, José Moutinho ${ }^{\mathrm{c}}$, Antonio M. G. Pinheiro ${ }^{\mathrm{a}}$ \\ ${ }^{a}$ Optics Center, Universidade da Beira Interior, Covilhã, Portugal \\ ${ }^{b}$ Instituto de Telecomunicações, Universidade da Beira Interior, Covilhã, Portugal \\ ${ }^{c}$ Faculty of Health Sciences, Universidade da Beira Interior, Covilhã, Portugal
}

\begin{abstract}
Breast ultrasound images have several attractive properties that make them an interesting tool in breast cancer detection. However, their intrinsic high noise rate and low contrast turn mass detection and segmentation into a challenging task. In this article, a fully automated two-stage breast mass segmentation approach is proposed. In the initial stage, ultrasound images are segmented using support vector machine or discriminant analysis pixel classification with a multiresolution pixel descriptor. The features are extracted using non-linear diffusion, bandpass filtering and scalevariant mean curvature measures. A set of heuristic rules complement the initial segmentation stage, selecting the region of interest in a fully automated manner. In the second segmentation stage, refined segmentation of the area retrieved in the first stage is attempted, using two different techniques. The AdaBoost algorithm uses a descriptor based on scale-variant curvature measures and non-linear diffusion of the original image at lower scales, to improve the spatial accuracy of the ROI. Active contours use the segmentation results from the first stage as initial contours. Results for both proposed segmentation paths were promising, with normalized Dice similarity coefficients of 0.824 for AdaBoost and 0.813 for active contours. Recall rates were $79.6 \%$ for AdaBoost and $77.8 \%$ for active contours, whereas the precision rate was $89.3 \%$ for both methods.
\end{abstract}

Keywords: Breast ultrasound, Image segmentation, Image analysis, Scale-space analysis

\section{Introduction}

Breast cancer is one of the major causes of mortality among women, particularly in developed countries. It stands as the leading cause of female death by cancer, and the fifth overall. In 2008, 1.384 million new cases were diagnosed and 458,000 deaths registered [1]. Nevertheless, a gradual decrease in breast cancer mortality has been noted, especially in developed or financially strong countries, where increased means of diagnosis are available. However, the number of cases worldwide continues to increase, and breast cancer is becoming the most prevalent cancer $[1,2]$. Therefore, there is a need for effective diagnostic tools enabling prevention, monitoring and early detection of new cases.

Different imaging techniques are frequently used for breast cancer screening and diagnosis, including

Email addresses: rafael.rodrigues@ubi.pt (Rafael Rodrigues), pinheiro@ubi.pt (Antonio M. G. Pinheiro) mammography, magnetic resonance imaging and ultrasound imaging [3]. Ultrasonography is a non-invasive, cost-effective and practically harmless technique that provides real-time diagnostic capability. Ultrasound can diagnose cysts with an accuracy near $100 \%$, which helps to limit unnecessary biopsies [4]. It is frequently used as a follow-up technique or as an adjunct to mammography in detection and diagnosis. Although mammography is currently the most widely used imaging method, breast ultrasound (BUS) imaging has been emphasized as a valuable tool for early cancer detection and diagnosis because of its attractive properties [5]. However, BUS images are typically characterized by speckle noise, shadows or other artifacts and poor edge definition, which are intrinsic to the imaging acquisition process and may result in a difficult and subjective analysis, even for experienced radiologists and oncologists (Fig. 1) [6, 7, 5]. Therefore, computeraided diagnosis (CADx) systems may become useful for both radiologists and oncologists. The development

Manuscript submitted to Ultrasound in Medicine $\mathcal{E}$ Biology

(C) 2015. This manuscript version is made available under the CC-BY-NC-ND 4.0 license.

[http://creativecommons.org/licenses/by-nc-nd/4.0/] 


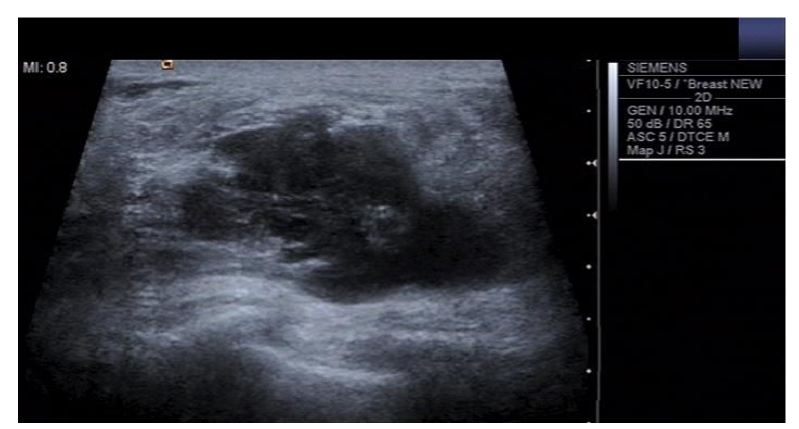

Figure 1: Breast ultrasound (BUS) sample image.

of such systems has attracted growing interest among researchers in this field.

The work described here focuses on the detection and segmentation of masses in BUS images. The images are pre-processed to deal with the constraints of typical ultrasound characteristics, and certain features obtained with the image processing techniques are used in the segmentation task. Typically, image segmentation is a difficult and complex task, which depends greatly on the type of image and may require large amounts of information to produce accurate and reliable results. However, under well-defined conditions, it is possible to obtain successful segmentation results, as described in [8], who performed facial recognition using an AdaBoost algorithm.

Several image segmentation methods have been proposed to respond to the challenging task of lesion segmentation in BUS images. Histogram thresholding is a fast and simple method that does not require training. It is one of the most often used segmentation techniques for monochromatic images. Histogram thresholding was used by Yap et al. [9], with relative success, to identify lesion candidates and delimit lesions in BUS images. Other researchers have applied histogram thresholding to pre-processed images. Horsch et al. [10] used median filtering and a Gaussian constraint function to reduce speckle prior to intensity thresholding. Chen et al. [11] used a median filter along with the negative of a bidimensional Laplacian filter, which was used to enhance the contrast of meaningful elements. Despite its limitations, when applied to images with unimodal histograms, histogram thresholding allows simultaneous detection of multiple masses, as described by Joo et al. [12]. However, noise sensitivity associated with intensity histograms may lead to inaccurate results. Adaptive thresholding was tested in Yeh et al. [13] and was highly correlated with manual segmentation. Also, the efficiency of the method in the presence of speckle noise was increased.

Model-based segmentation techniques have also been tested on BUS images. In [14], a Markov random field was applied with the focus on the adaptive features of the algorithm, which was controlled by a weighting function. The algorithm estimated iteratively the class parameters and assigned a class label to each pixel, considering local and global statistical measures. In another work [15], maxima a posteriori in estimating the distortion field, which was followed by a multiplicative model and pixel labeling. The application of model-based algorithms is noise resistant and has some potential in BUS segmentation. Nevertheless, imaging models tend to break down in the presence of shadows, and the processes may become rather complex and time consuming [3].

Other model-based approaches include deformable models such as active contours and level sets. Snake active contours have been applied to BUS segmentation with good results. In [16], parametric active contour models such as gradient vector flow and balloon were used in BUS mass segmentation, after pre-processing with median filtering and histogram equalization. A segmentation refinement stage was designed, integrating curvature information or even empirical knowledge to improve the initial result. Another method described by Madabhushi and Metaxas [4] relies on the automatic definition of seed points based on empirical knowledge given by radiologists. Region growing was then applied to obtain an initial contour. Image and texture information was used to classify the pixels, and the boundary points found with a directional gradient served as an initial contour for an active contour model, which used the directional gradient as a stopping criterion.

A level set model was applied to the segmentation of lesions in BUS [17], yielding better results when compared with active contours. The initial contour was obtained through binary thresholding after applying the modified curvature diffusion equation (MCDE) to remove noise and enhance the image contours. In [18], the ultrasound image was first processed using anisotropic diffusion filtering. Then, an initial contour for the level set segmentation was obtained combining the stick method with binary thresholding. Despite the good segmentation results and low noise sensitivity, segmentation using deformable models, such as active contours or level set models, has a number of significant drawbacks with respect to the automatic definition of an appropriate initial contour as well as the high sensitivity to local minima. Also, the convergence of the active deformation process may be computationally heavy. 
In [19], a modified watershed algorithm was used for semi-automatic contour extraction of BUS lesions. Morphological operations were first applied to obtain more accurate contours and prevent typical watershed oversegmentation [20]. The watershed transform was also used in [5] for automatic lesion detection. Images were preprocessed by mean filtering and fuzzy logic histogram thresholding. In both cases, watershed segmentation achieved high accuracy, similar to that of manual segmentation.

A k-means algorithm was tested in [21] to achieve the segmentation of BUS images. The authors classified tissues using an adaptive method based on texture information. Even though the algorithm is simple and effective, the results were dependent on system parameters and a major problem might have arisen from eventual similarities between the mass and shadows or other artifacts in the image, which could lead to inclusion in the wrong cluster of pixels.

Classification algorithms have been widely considered as segmentation alternatives for a number of image modalities. In the specific case of BUS, several studies have used support vector machines (SVMs) and neural networks (NNs) to obtain lesion contours. In [22], the authors successfully combined wavelet analysis of the image with an error retro-propagation $\mathrm{NN}$, using contrast variance and autocorrelation as inputs. Another study [23] classified BUS images employing a watershed segmentation algorithm along with a NN trained using texture descriptors. A Bayesian NN with five hidden layers was also tested in BUS image segmentation in [24]. Texture, gradient and acoustic information of the images was retrieved to train the $\mathrm{NN}$, which was used to validate candidate regions obtained with a region-growing algorithm, starting from points of interest defined using the image gradient. However, the method proved to be unreliable, especially where lesions were not uniform. In [25], a similar seed point approach was used, along with pixel classification using NN. Multidomain features such as intensity, texture, phase in max-energy orientation and radial distance were combined. This method yielded interesting segmentation results in BUS. Su et al. [26] applied self-organizing maps, using textural local information, to obtain an initial contour. This outline would later be segmented with active contours, culminating in a fully automated method with good accuracy. Combining SVMs with textural information, Liu et al. [27] proposed a robust, high-precision method for mass segmentation in BUS. Although the use of classifiers for targeting ultrasound images has had promising results, the training required and the selection

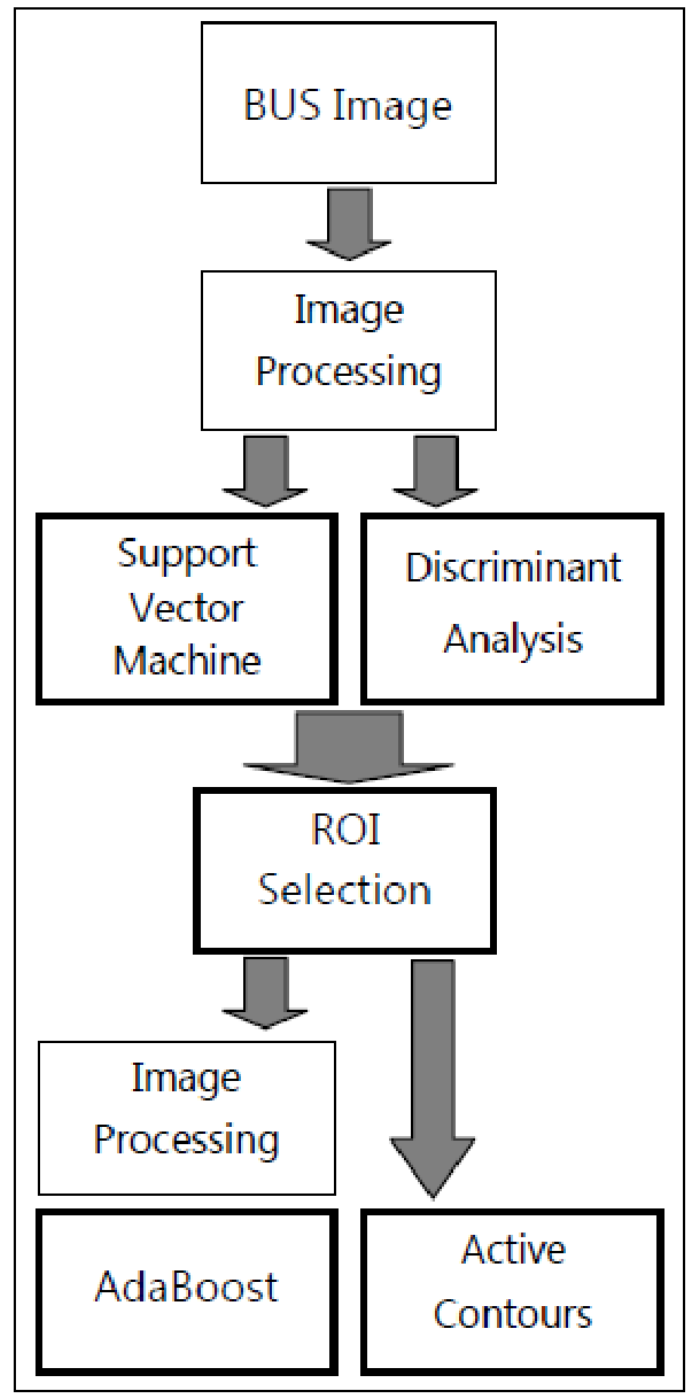

Figure 2: Global workflow for the proposed approach. $\mathrm{BUS}=$ breast ultrasound; $\mathrm{ROI}=$ region of interest.

of an appropriate set of features for its application can make the task complicated and time consuming [23].

Despite the growing range of methods for BUS lesion segmentation, achieving a fully automated optimal solution is still a challenging task. Some of the aforementioned segmentation methods require manual selection of an initial region of interest (ROI) to obtain a proper segmentation $[21,19,16]$. In addition, as previously discussed, the intrinsic properties of ultrasound images are not compatible with most standard segmentation techniques or make the segmentation task too complex with respect to the results obtained. In this work, the proposed method configures a two-step 
fully automated approach (Fig. 2). The first stage uses a SVM [28] or discriminant analysis (DA) [29] to obtain an initial segmentation. For the second stage, two different methods were tested. An AdaBoost classifier or active contours are used to improve the spatial accuracy. Final results indicated the correct location of the lesions in all cases. In terms of segmentation accuracy, the best result is characterized by a Dice coefficient of 0.824 with a computation time of $12 \mathrm{~s}$.

\section{Methods}

\subsection{Data collection}

Images were collected at Hospital da Cova da Beira in Covilhã, Portugal, by an experienced oncologist using a LOGIQ Book XP (GE Healthcare, Little Chalfont, UK) with a linear probe. All subjects involved in the study provided informed written consent and were explained the purpose of the experiment. The study followed the tenets of the Declaration of Helsinki by the World Medical Association and was approved by the institutional review board.

\subsection{Feature extraction}

To classify BUS images, SVM and DA classification algorithms were applied, using a pixel descriptor with five different features. The features considered were the non-linear diffusion [30] of the original BUS image, two bandpass FIR filter [31] outputs with distinct bandpass intervals and two different scale-space mean curvature measures $[32,33]$. The main goal of defining such a set of features was to combine both texture and graylevel information. Texture features have been found to effectively characterize and distinguish lesions from tissue regions and shadows [4]. The non-linear diffusion of the images $\left(I_{d i f}\right)$ was obtained with the method described by [30]. As discussed in the same work, an adaptive computation method for the constant $\mathrm{K}$ was defined. The gradient histogram was computed in each iteration, and $\mathrm{K}$ was set to $90 \%$ of its integral. Conduction coefficients of the diffusion scheme were computed using the Charbonier conductivity function [34]:

$$
g(\nabla I)=\frac{1}{1+\left(\frac{\|\nabla I\|}{K}\right)^{2}}
$$

where $\nabla I$ is the intensity gradient. The use of this function intends to privilege the conservation of wide regions of the image while promoting intra-region smoothing, which directly reduces the influence of noise. The resulting diffusion images considered in the pixel descriptors were obtained after 60 iterations, with a fixed constant $\lambda=1 / 8$ (Fig. 3(b)).

With respect to bandpass filtering, two different results were computed for each image: $I_{b p 1}$ using a lower bandpass interval $(0: 031 \pi<\omega<0.056 \pi)$, and $I_{b p 2}$, using a higher bandpass interval $(0: 056 \pi<\omega<$ $0.1 \pi$ ), both included in the pixel descriptors (Fig. 3(c) and (d)). The filters were obtained using the McClellan transformation over 1-D FIR filters with $N=150$ [31]. Both bandpass filters provide valuable information on region transitions, simultaneously rejecting most of the highfrequency noise components that degrade the images.

Finally, the pixel descriptor contained information extracted from yet another image processing technique, which produces effective features for the proposed classification scheme. Mean curvature measures were taken on different scales. The scale of the original image varies on Gaussian filtering [33]. Despite its poor spatial definition, Gaussian smoothing also effectively reduces high-frequency noise components [32] that degrade intra-region stability of ultrasound images and compromise the definition of the edges.

Gaussian smoothing was performed on the original image, over a series of iterations, using a $5 \times 5$ filter $\left(F_{\text {gauss }}\right)$ with:

$$
\sigma^{2}=\frac{\sqrt{2}}{2}
$$

Filter size and standard deviation of the filter were constant for all iterations.

The curvature was computed in two different scales $(t=200$ and $t=300)$. For both scales, the principal curvatures $L_{x x}$ and $L_{y y}$ were computed. These correspond to the second-order directional derivatives of the Gaussian smoothed image $I_{\text {gauss }}$ and were obtained numerically. Initially, the gradients $L_{x}$ and $L_{y}$ are computed with the equations:

$$
\begin{aligned}
& L_{x}(i, j)=I_{\text {gauss }}(i, j)-I_{\text {gauss }}(i+1, j) \\
& L_{y}(i, j)=I_{\text {gauss }}(i, j)-I_{\text {gauss }}(i, j+1)
\end{aligned}
$$

Then, the second-order gradients are obtained in a similar manner:

$$
\begin{aligned}
& L_{x x}(i, j)=L_{x}(i, j)-L_{x}(i+1, j) \\
& L_{y y}(i, j)=L_{y}(i, j)-L_{y}(i, j+1)
\end{aligned}
$$




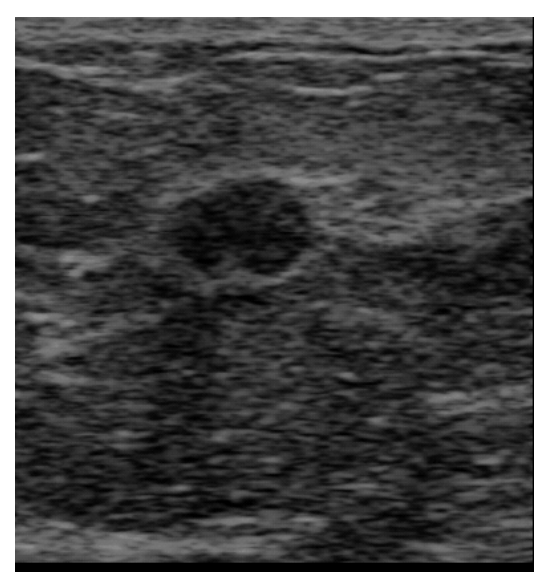

(a)

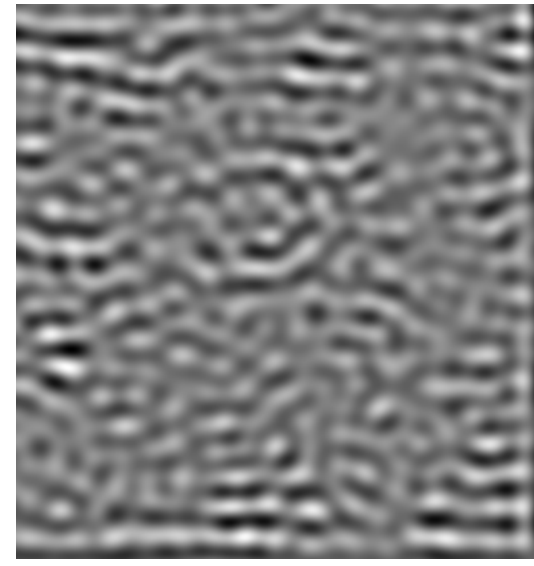

(d)

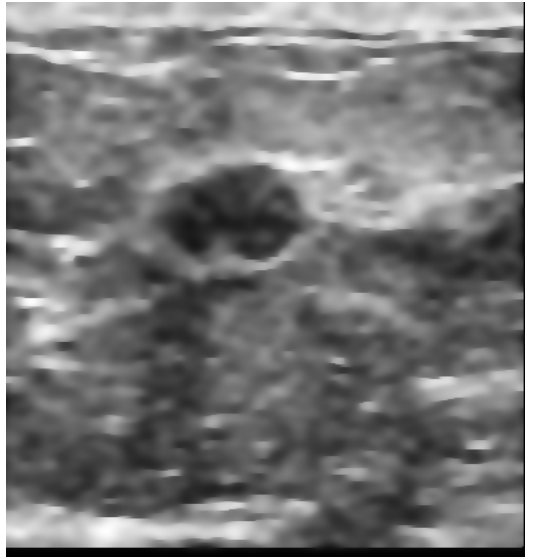

(b)

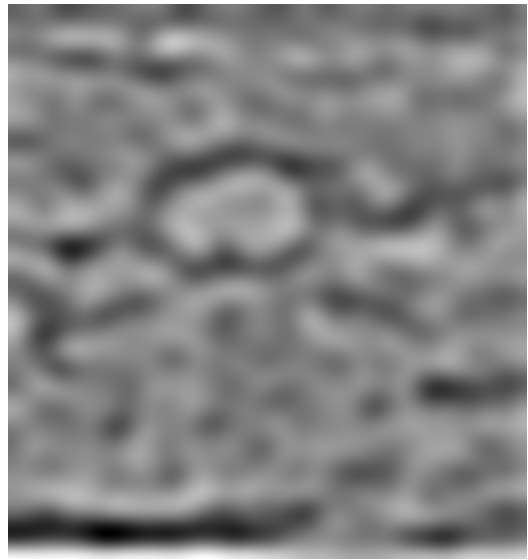

(e)

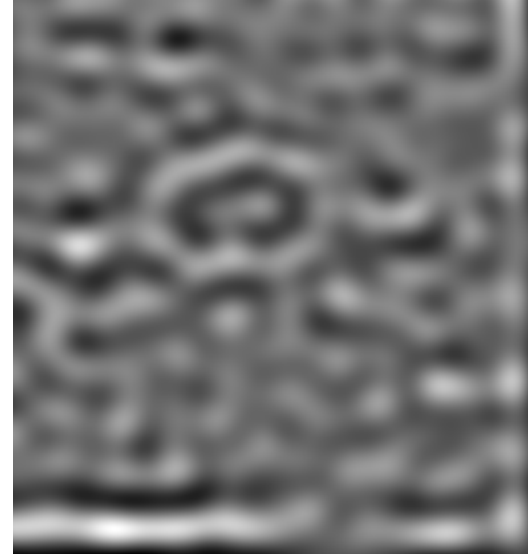

(c)

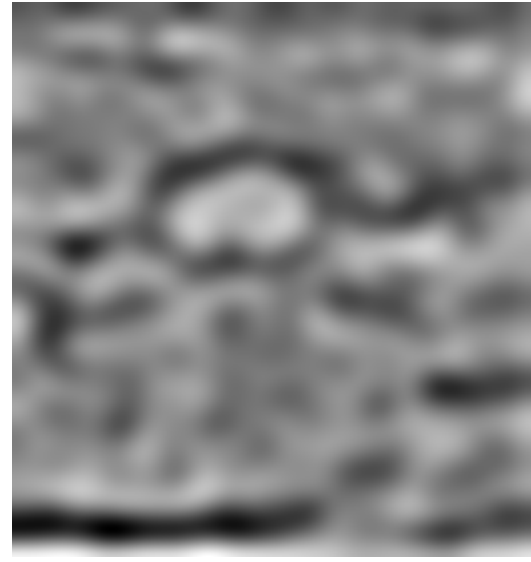

(f)

Figure 3: Original image and pixel descriptor components. (a) Original image. (b) Non-linear diffusion $(t=60)$. (c) Lower bandpass filtering. (d) Higher bandpass filtering. (e) Scale-space curvature $(t=200)$. (f) Scale-space curvature $(t=300)$.

Finally, the mean curvature $\mathrm{H}$ is given by the arithmetic mean of the principal curvatures of the image [33]:

$$
H=\frac{L_{x x}+L_{y y}}{2}
$$

The mean curvature presents local maxima in inflexion points localizations. Thus, the curvature yields a good representation of region transitions. The Gaussian smoothing of the images, prior to computation of the mean curvature, reduces the influence of noise, which would degrade the curvature results as it represents a mapping of the intensity variation on the image [33].

Fine parameter setting was based on experimentation. Also, the chosen features were normalized to address the question of data dependency. Figure 3 illustrates output examples of the aforementioned image processing methods.

\subsection{Initial segmentation using SVM and DA classifiers}

In the initial segmentation stage, the training data set for the SVM and the DA classifiers comprised 654 randomly selected pixels from two sonograms of two distinct patients. These training samples were collected from two strips defined around the true binary segmentation masks, manually defined by an oncologist. The main goal of this selection strategy was to obtain a representative group of samples near the region transitions, which were assumed to promote an easier and less ambiguous tissue differentiation. Considering the small available database, it was essential to implement a training scheme that could yield effective models without the selection of a large training data set. Furthermore, the aim of selecting pixels from restricted and specific areas, rather than the whole image, was to reduce the noise sensitivity of the method. 
To define these strips, the true segmentation mask was dilated and eroded using binary morphological operations and different-sized circular structuring elements. A 2-pixel-wide strip containing positive labeled pixels was created surrounding the inner limits of the mask (green pixels in Fig. 4), and a strip of negative labeled pixels of the same width surrounded the outer limits of the mask (red pixels in Fig. 4). From the obtained strips obtained, $20 \%$ of the pixel locations were randomly selected to form the training set with 313 positive pixels and 341 negative pixels. Because the strip outside the mask is larger, the number of resulting negative samples is also larger.

Pixel classification was performed with a SVM classifier using the Linear, Quadratic, Polynomial, Radial Basis Function and the Multilayer Perceptron kernels, and also a DA classification algorithm using the types Linear, Quadratic, Diagonal Linear, Diagonal Quadratic and Mahalanobis Distance.

\subsection{ROI selection}

This stage includes a set of simple heuristic operations and plays a determinant role in the proposed segmentation scheme. Use of these operations over the initial SVM or DA classification results greatly reduces the number of misclassified pixels. Also, this stage yields the initial contours used in the subsequent segmentation steps.

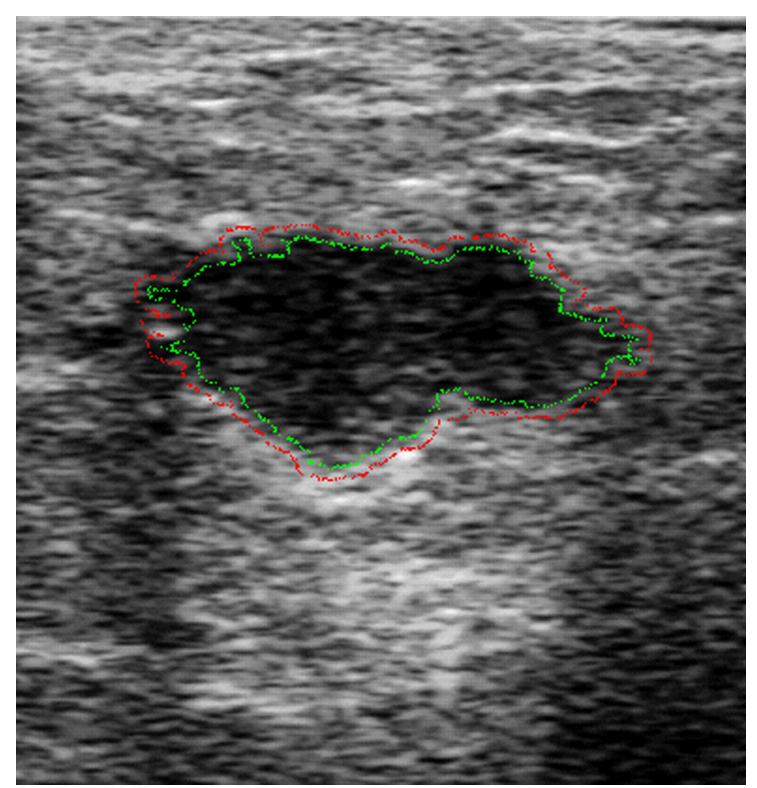

Figure 4: Training data selection.
Initially, a filling operation was performed on the image pixel classification results, suppressing holes inside the ROI. Given the strategy used in SVM and DA training, where edge pixels were selected to construct the training data set, the holes within segmented areas were expected.

Furthermore, foreground regions directly connected to the image borders were suppressed (Fig. 5(c)). It is assumed that these regions were not part of the ROI, often resulting from artifacts or shadows in the ultrasound exam. However, these regions are likely to be misclassified as ROIs because of the similar grayscale intensity values, when compared with the lesions. This simple step yielded binary images containing the ROI and, in most images, other small isolated undesired regions.

To further reduce the impact of small misclassified regions, two different heuristic methods were tested. Morphological opening was performed over the binary images in the first approach, using an 8-pixel-radius circular structuring element (Fig. 5(d)). The regions that were not suppressed by the opening operation were retrieved in their original shape prior to the opening operation (Fig. 5(e)), providing more accurate contours. In the second approach, only the largest foreground area was selected and any other region was suppressed (Fig. 5(f)).

Extraction of a single ROI is desirable to use the segmentation refining techniques. Using morphological opening with a fixed structuring element radius may either allow misclassified areas in its output or suppress small true positive areas. With attention to these limitations, the largest area selection method was chosen.

To simplify analysis of the segmentation refinement methods, given that 10 different classifiers were tested for the initial segmentation, the maximum accuracy criterion was applied to select only one image.

\subsection{Segmentation refinement using AdaBoost classifier}

The AdaBoost algorithm [35] uses a weak classifier that simply establishes a threshold for each data dimension according to a distribution $D_{n}$, dividing data samples in two classes. This classifier is called iteratively and the threshold is adapted to minimize the classification error. The training phase yields a model that defines the sequence of data dimensions and thresholds that minimizes the training error, measured against the segmentation masks of the training data. In each training iteration $n$, a weight factor $\alpha_{n}$ is assigned to the hypothesis given by the weak classifier 


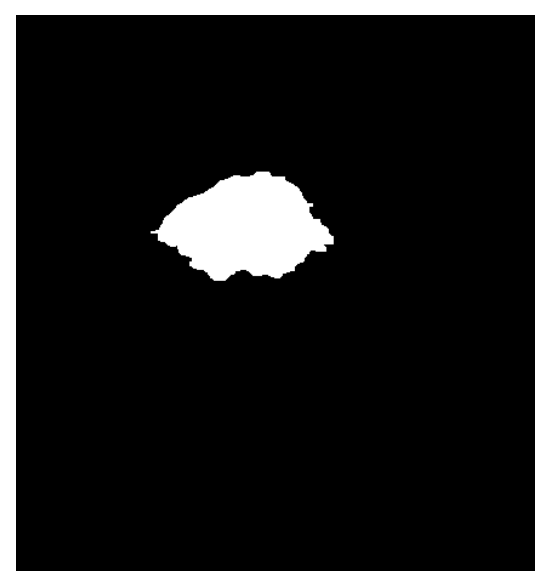

(a)

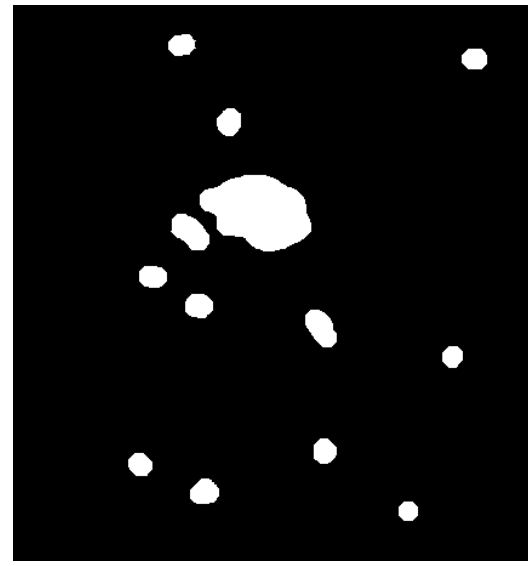

(d)

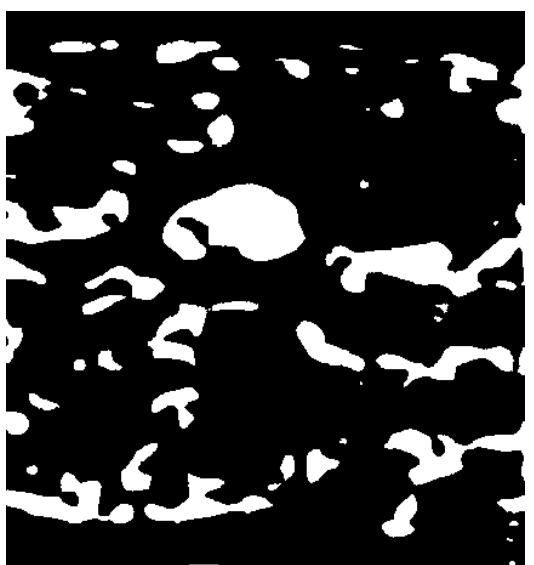

(b)

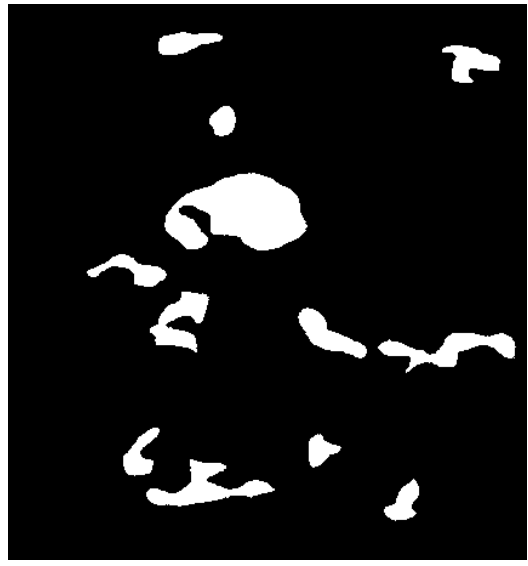

(e)

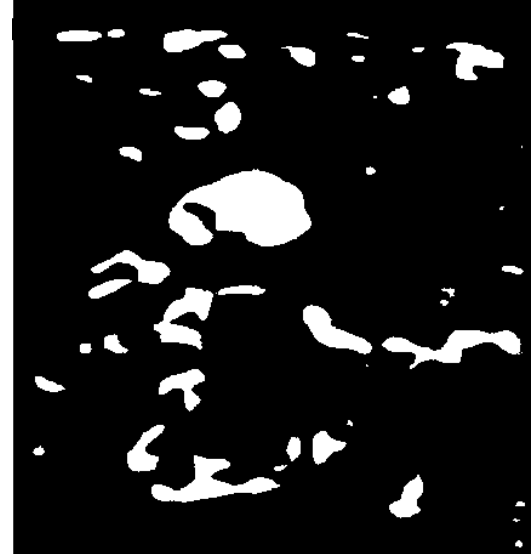

(c)

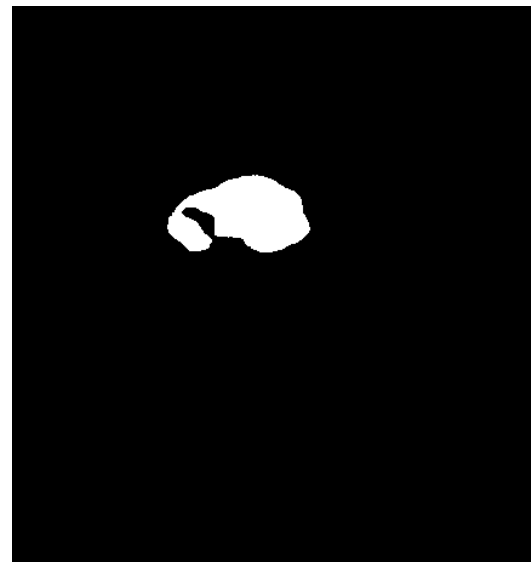

(f)

Figure 5: Region of interest selection. (a) True segmentation mask. (b) Classifier result. (c) Border cleaning. (d) Opening. (e) Opening selection. (f) Largest area selection.

( $h_{n}: X \rightarrow Y \in\{-1,1\}$ for binary classification). Then, the distribution $D_{n}$ is updated with respect to $\alpha_{n}$. The weight factor $\alpha_{n}$ varies inversely with the error $\epsilon_{n}$, which is given by

$$
\epsilon_{n}=\operatorname{Pr}_{i \sim D_{n}}\left[h_{n}\left(x_{i}\right) \neq y_{i}\right]=\sum_{i: h_{t}\left(x_{i}\right) \neq y_{i}} D_{n}(i)
$$

This causes misclassified examples to have greater preponderance in the next iteration and promotes faster error convergence and minimization as the algorithm focuses on harder examples. The resulting AdaBoost model is then applied in the classification phase, and the final result $\mathrm{H}$ is a weighted majority vote considering all the weak hypotheses.

The features used to define the pixel descriptor employed for the segmentation refinement were similar to those applied previously, but with distinct parameterizations. Focusing on the ROI area directly reduces the influence of noise and other objects that might compete wit the masses. Thus, lower-scale features were chosen, which offer better spatial accuracy properties when compared with the higher scales used in the initial stage.

The AdaBoost classifier was trained and applied with 200 iterations. The number of iterations was approximated experimentally so that $\epsilon_{n} \leq 0.05$ and the difference $\left|\epsilon_{n-1}-\epsilon_{n}\right| \leq 0.001$ in five consecutive iterations to confirm error convergence to a stability level [35].

The training data set was extracted from the same BUS images used in SVM and DA training, and training sample selection followed a similar logic. The negative training data set was formed by the random selection of $50 \%$ of the pixels located in a strip defined around the manual segmentation mask. As for the positive training data set, $20 \%$ of the true positive mask pixels were extracted. The resulting training data set included 


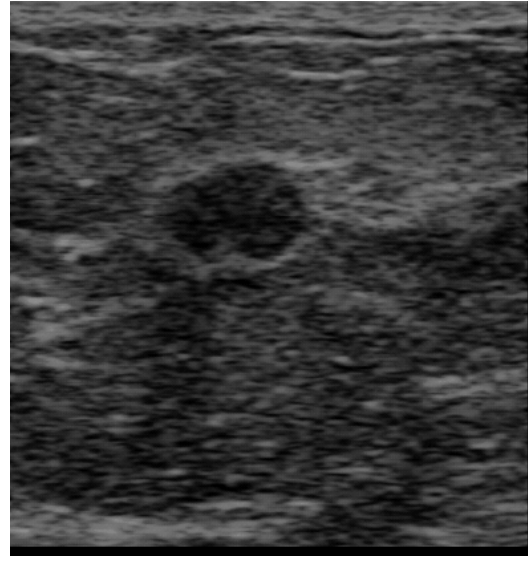

(a)

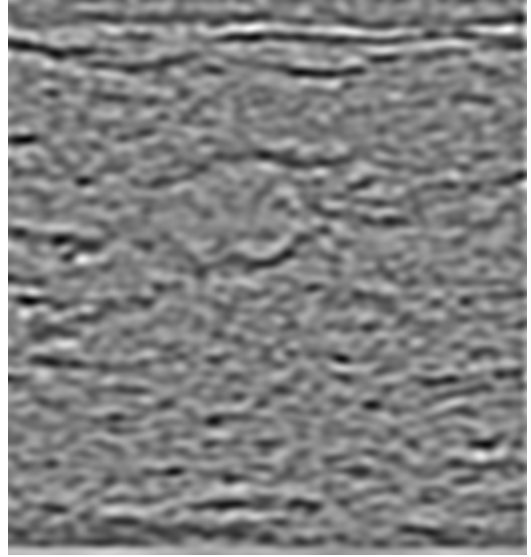

(c)

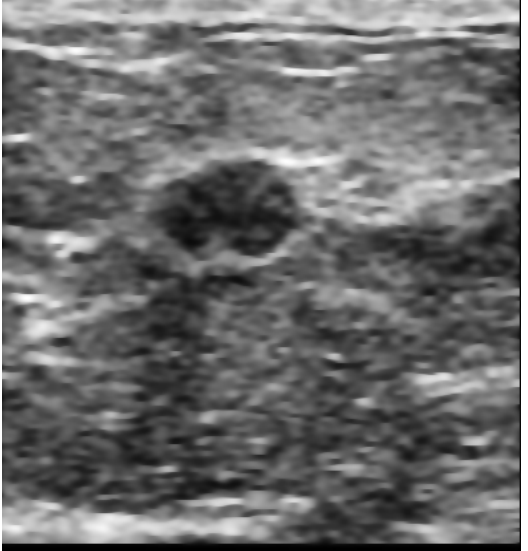

(b)

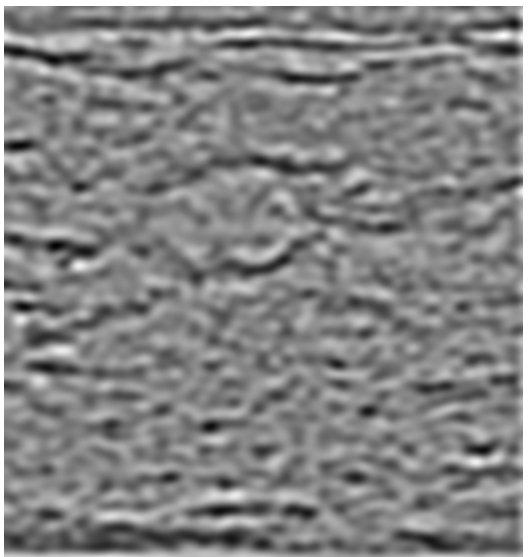

(d)

Figure 6: Pixel descriptor components for AdaBoost classifier. (a) Original image. (b) Non-linear diffusion $(t=20)$. (c) Scale-space curvature $(t=20)$. (d) Scale-space curvature $(t=30)$.

2,429 pixels, from which 1,392 were positive and 1,037 were negative. The increase in the number of training samples used in comparison with those considered in the previous classification stage seeks to reinforce the segmentation refinement framework, exploiting the fact that AdaBoost is computationally lighter than SVM and DA algorithms.

The pixel descriptor included curvature measures at scales $t=20$ and $t=30$ and non-linear diffusion of the original image after 30 iterations, with the same parameterization used in the first step (Fig. 6). Similarly to the preceding stage, parameter setting was based on experimentation, and the features were normalized. Bandpass filtering was omitted from this stage as it did not contribute to the robustness of the AdaBoost classifier.

Moreover, the classifier was applied to a dilated region around the initial ROI area. Only pixels inside this area were considered in the classification task. The pixels outside this area are automatically considered as background. For classification of the images, the initial ROI segmentation masks were dilated with a circular structuring element with radius 8 . The size of the structuring element was defined based on experimentation, to keep a balanced trade-off between selection of the largest possible area and maintenance of the focus on the ROI.

\subsection{Segmentation refinement using active contours}

The second path proposed for segmentation refinement of the masses relies on active contours without edges. The applied algorithm implements the method described in [36], which focuses on minimization of the energy equation: 


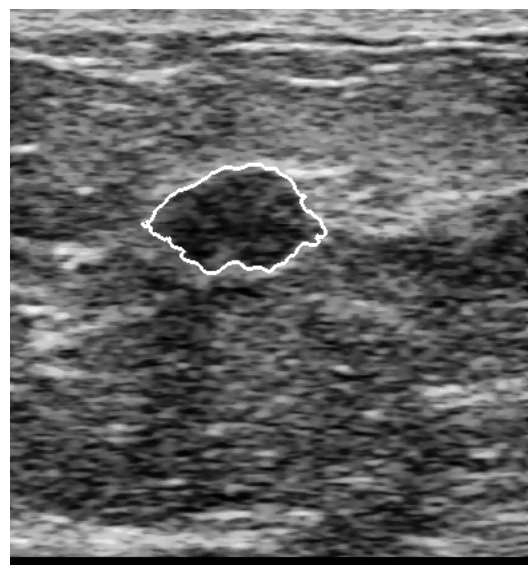

(a)

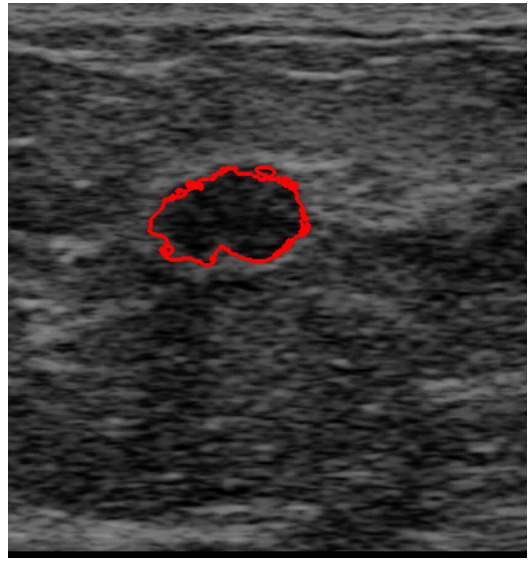

(c)

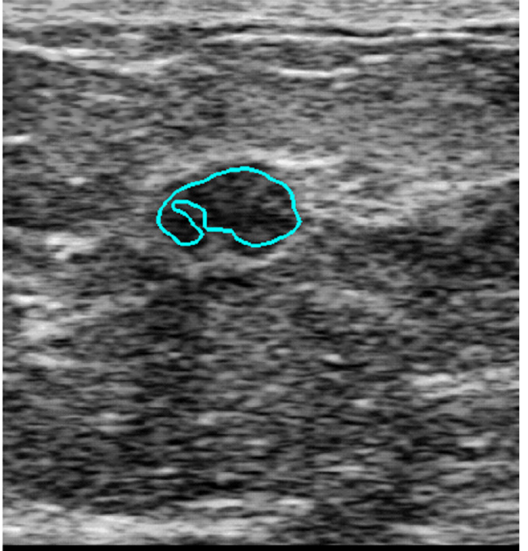

(b)

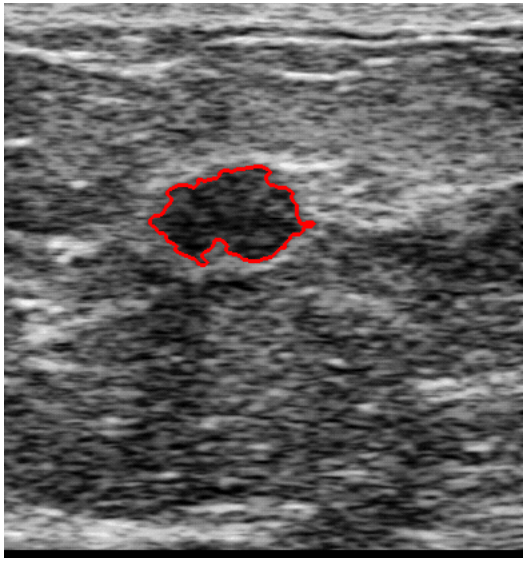

(d)

Figure 7: Final segmentation results I. (a) Manual segmentation. segmentation.

$$
F_{1}(C)+F_{2}(C)=\int_{C_{i}}\left|u_{0}-c_{1}\right|^{2} d x d y \int_{c_{o}}\left|u_{0}-c_{2}\right|^{2} d x d y
$$

Initial segmentation results were directly used to define an initial contour for the active contour algorithm, which was applied in 100 iterations. Similarly to the preceding stage, the output of the algorithm was submitted to the selection of the largest area object, to eliminate small non-relevant objects that might result from fragmentation of the main contour, yielding the final segmentation results.

\subsection{Experimental validation}

The proposed segmentation scheme was tested on 44 breast sonograms from 29 patients. The database included only images with masses, with no distinction between malignant and benign tumors, as it did not serve the purpose of this work. Despite being rather small, the testing database was diversified, considering images severely degraded by speckle noise and shadows. Images from the same patient were obtained at different stages of illness, providing different content. In all cases, the whole sonogram was used as input to the algorithm. Manual tumor delimitation by an experienced oncologist was used as ground truth. The Dice similarity coefficient was used to evaluate the performance (overlap). Accuracy, recall and precision measures were also considered to assess global algorithm performance.

\section{Results}

The overall segmentation performance measures are listed in Table 1. Initial segmentation results indicated 


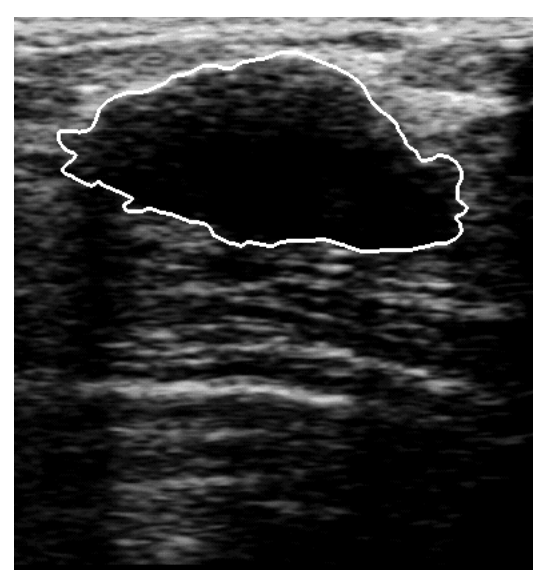

(a)

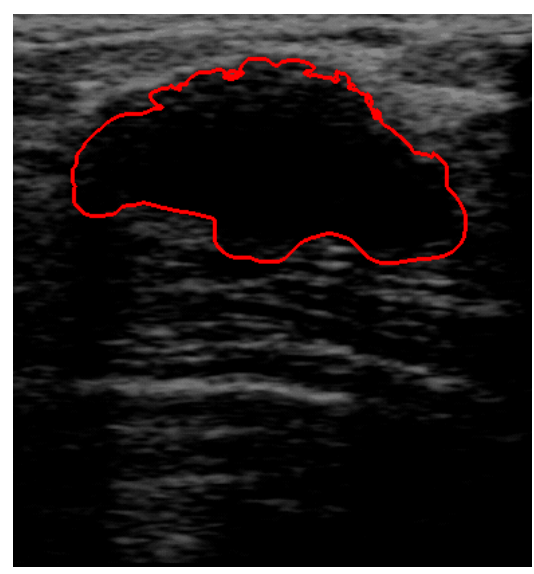

(c)

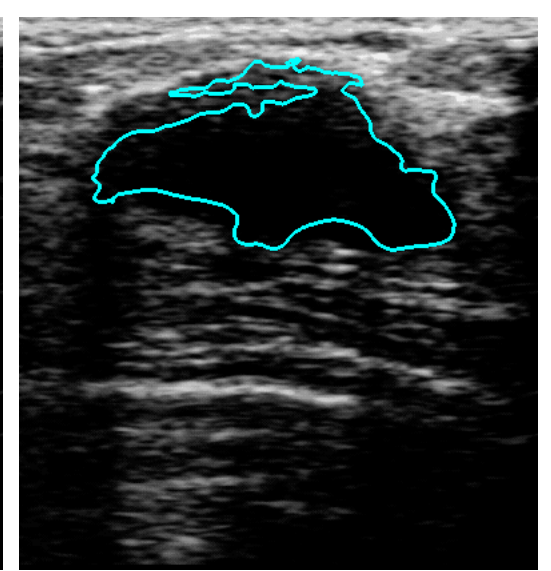

(b)

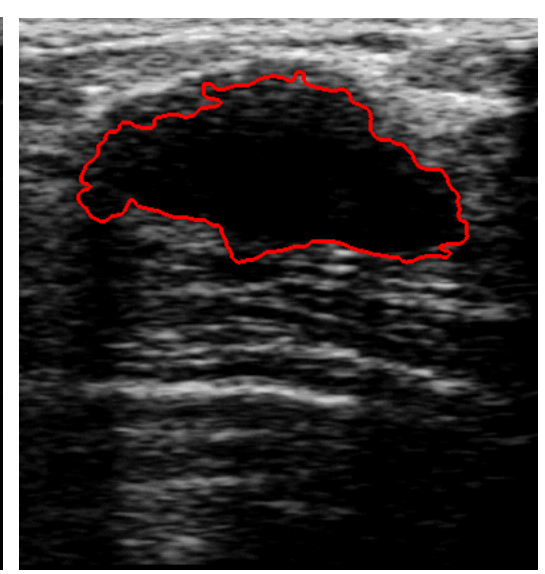

(d)

Figure 8: Final segmentation results II. (a) Manual segmentation. (b) Initial segmentation. (c) AdaBoost segmentation. (d) Active contour segmentation.

high precision $(92.4 \%)$, but a relatively low recall rate $(68.1 \%)$ and overlap coefficient (0.690). Both AdaBoost and active contour refining methods achieve better overlap and recall results than the initial segmentation algorithm, indicating that the spatial accuracy of the detected masses is improved. In a direct comparison of the two segmentation refinement methods, AdaBoost improves the normalized overlap coefficient by 0.134

Table 1: Segmentation performance measures

\begin{tabular}{lccc}
\hline & & & \\
& Initial & AdaBoost & Active contours \\
\hline Accuracy & $97.3 \%$ & $97.7 \%$ & $97.5 \%$ \\
Recall & $68.1 \%$ & $79.6 \%$ & $77.8 \%$ \\
Precision $_{\text {Dice }_{\text {coef }} \text { (overlap) }}$ & $92.4 \%$ & $89.3 \%$ & $89.3 \%$ \\
\hline
\end{tabular}

$\left(\right.$ Dice $\left._{\text {coef }}=0.824\right)$, whereas active contours improve this measure by $0.123\left(\right.$ Dice $\left._{\text {coef }}=0.813\right)$. The recall rate was improved by $11.5 \%$ using AdaBoost (recall $=$ $79.6 \%$ ) and $9.7 \%$ with active contours (recall $=77.8 \%$ ).

The average running time for segmentation of a single sonogram was $12 \mathrm{~s}$ (initial segmentation and refinement) on an Intel Core i7-2600 CPU (3.40 GHz) using MATLAB R2013a (The MathWorks, Natick, MA, USA) without code optimization.

\section{Discussion}

The relatively low recall rates are due mostly to the loss of spatial accuracy resulting from high nonlinear diffusion and curvature scales. On the other hand, using lower scales on the entire image could increase the segmentation noise and complicate correct 


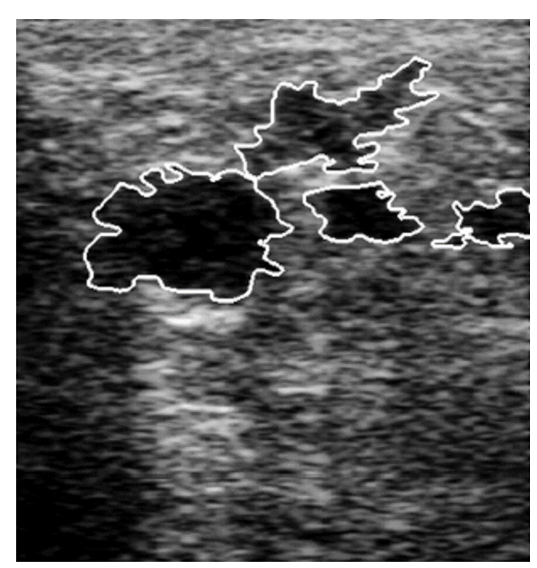

(a)

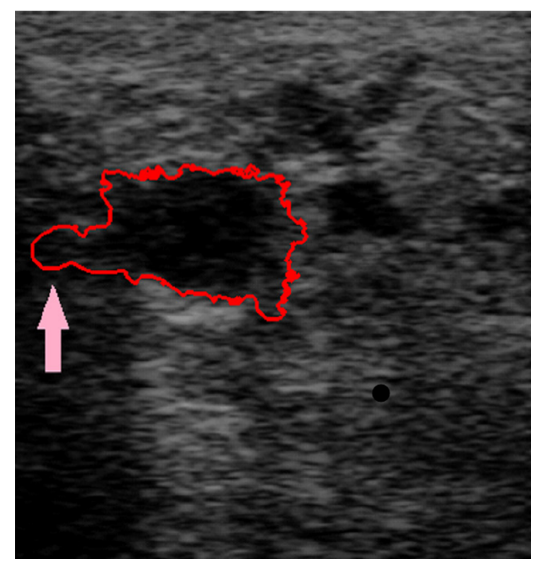

(c)

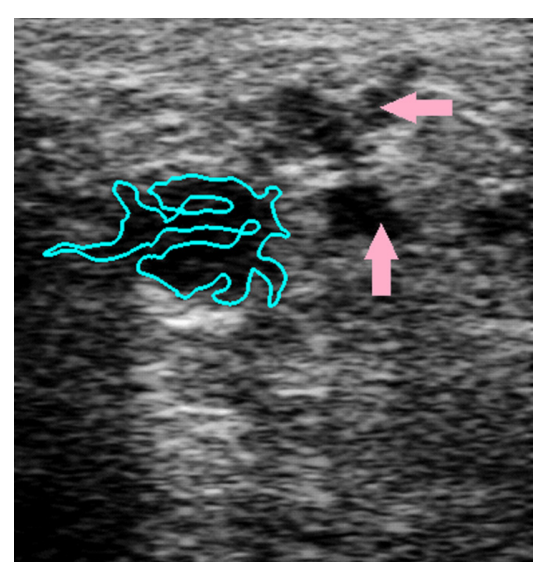

(b)

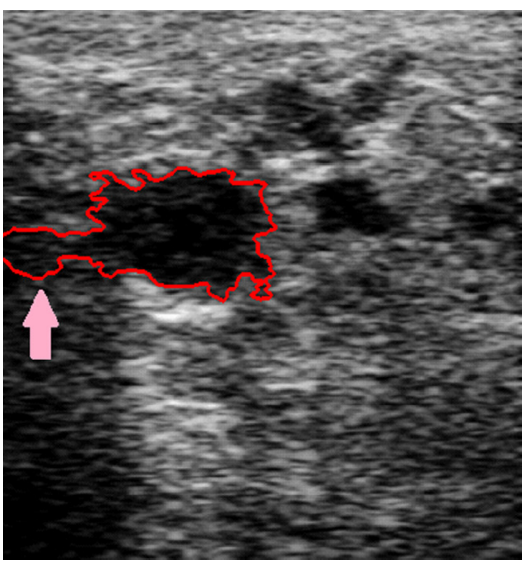

(d)

Figure 9: Final segmentation results III. (a) Manual segmentation. (b) Initial segmentation.

(c) AdaBoost segmentation.

(d) Active contour segmentation.

detection of the ROI. For this reason, the segmentation refinement methods were used in the proposed scheme. In addition, manual segmentation masks used to evaluate segmentation performance tend to define larger contours because of the poor edge definition of the images, which also has an influence on recall rates.

In this database, some of the images revealed two or more masses (Fig. 9 (a)). The largest area criterion used to select the ROI directly reduces the efficiency of the algorithm in cases with multiple ROIs, because the detection of a second mass is deliberately ignored (Fig. 9 (b)). Table 2 summarizes the results obtained by considering only the largest mass in each ground truth image. The precision results remain unchanged when compared with Table 1 because true positive and false positive pixels are exactly located in the ROI area.

In some cases, the initial segmentation outputs are much smaller than true positive masks (Fig. 7 (b)).
This may affect some AdaBoost segmentations because of the fixed size of the structuring element that dilates the initial mask, meaning that it may not be possible to obtain a full segmentation of the ROI. This shortcoming of the AdaBoost method might be improved, defining several segmentation cycles that sequentially dilate the output of the previous segmentation. However, a stopping criterion would be necessary to prevent deviation from the ROI in cases where the initial segmentation contains false positives (Fig. 9 (c)). On the other hand, this constriction focuses AdaBoost on the ROI detected initially by the SVM or DA classifiers.

Active contours are adjusted to the mass contour from the inside (Fig. 8 (b)), due to the properties of most initial segmentations. In such cases, the algorithm yielded good results. However, in some examples, the initial contour is influenced by false positives in the initial segmentation. Then, the contour may depart from 
Table 2: Segmentation performance measures considering only the largest mass from the ground truth

\begin{tabular}{lcc} 
& AdaBoost & Active contours \\
\hline Accuracy & $97.8 \%$ & $97.6 \%$ \\
Recall & $80.1 \%$ & $78.2 \%$ \\
Precision & $89.3 \%$ & $89.3 \%$ \\
Dice $_{\text {coef }}$ (overlap) & 0.831 & 0.820 \\
\hline
\end{tabular}

the ROI because of low contrast resolution in edges. Also, the lack of a limiting region exposes the active contour to shadows near the ROI (Fig. 9(d)). These deviations directly increase false positives and lower precision.

Both proposed pathways achieve promising segmentation results, even in images containing large regions of shadows or other tissues (Fig. 8(c) and (d)), despite the relatively low recall rates. The algorithm configures a fast and fully automated solution for lesion segmentation where the whole sonogram is taken as input. False negatives may influence the clinical decision, leading to inaccurate diagnosis in terms of tumor size and shape. Although the fine segmentation boosts the initial results, the improvement of training sample selection in the first stage (SVM or DA classification) or the introduction of new features might play an important role in the overall performance of the method. The implementation of an appropriate stopping criterion for the active contour algorithm might also be of interest.

In this research, parameter selection was performed on the basis of empiric evidence resulting from a long experimental process. Automatic parameter selection is regarded as an objective for future research. Furthermore, future work might include testing the proposed methods on a larger BUS database, as well as analyzing the influence of lesion type in the results. The absence of a benchmark BUS database hinders that task. Also, comparison of the performance of this method with that of other known methods is an immediate objective to further assess the performance of the system.

\section{Conclusions}

Breast cancer has attracted the interest of the scientific community in recent years. Early breast cancer detection may play a determinant role in the reduction of mortality associated with this pathology. The pace of research toward the development of effective CADx systems has been increasing, yet it still remains a complex and challenging task.

In this work, a two-step segmentation method was applied to the segmentation of masses in a BUS database. This algorithm integrates a variety of techniques frequently tested in image segmentation. Empirical information was also used to complement the algorithm. Despite the heuristic nature of the ROI selection methods, these steps assume an essential role in the automation of the process, which represents its main advantage.

\section{Ackowledgements}

The authors acknowledge the support from Universidade da Beira Interior, Covilhã, Portugal, and FCT Project PEst-OEFIS/UI0524/2014. This research was supported by a grant of the Instituto de Telecomunicações (CENTRO-07-ST24FEDER-002022) in the context of the project DERMCLASS: Feature Extraction and Artifact Removal in Dermoscopic Images.

\section{References}

[1] C. DeSantis, M. M. Center, R. Siegel, A. Jemal, Breast cancer facts \& figures 2009-2010, American Cancer Society (2010).

[2] M. Sant, S. Francisci, R. Capocaccia, A. Verdecchia, C. Allemani, F. Berrino, Time trends of breast cancer survival in Europe in relation to incidence and mortality, International Journal of Cancer 119 (2006) 2417-2422.

[3] H.-D. Cheng, J. Shan, W. Ju, Y. Guo, L. Zhang, Automated breast cancer detection and classification using ultrasound images: A survey, Pattern Recognition 43 (2010) 299-317.

[4] A. Madabhushi, D. N. Metaxas, Combining low-, high-level and empirical domain knowledge for automated segmentation of ultrasonic breast lesions, IEEE Transactions on Medical Imaging 22 (2003) 155-169.

[5] L. Zhang, Y. Ren, C. Huang, F. Liu, A novel automatic tumor detection for breast cancer ultrasound images, in: 2011 Eighth International Conference on Fuzzy Systems and Knowledge Discovery (FSKD), volume 1, IEEE, pp. 401-404.

[6] J. A. Noble, D. Boukerroui, Ultrasound image segmentation: a survey, IEEE Transactions on Medical Imaging 25 (2006) 9871010.

[7] C. M. Sehgal, S. P. Weinstein, P. H. Arger, E. F. Conant, A review of breast ultrasound, Journal of Mammary Gland Biology and Neoplasia 11 (2006) 113-123.

[8] P. Viola, M. Jones, Rapid object detection using a boosted cascade of simple features, in: Proceedings of the 2001 IEEE Computer Society Conference on Computer Vision and Pattern Recognition (CVPR 2001), volume 1, IEEE, pp. I-I.

[9] M. H. Yap, E. A. Edirisinghe, H. E. Bez, A novel algorithm for initial lesion detection in ultrasound breast images, Journal of Applied Clinical Medical Physics 9 (2008) 181-199.

[10] K. Horsch, M. L. Giger, L. A. Venta, C. J. Vyborny, Computerized diagnosis of breast lesions on ultrasound, Medical Physics 29 (2002) 157-164. 
[11] D.-R. Chen, R.-F. Chang, Y.-L. Huang, Computer-aided diagnosis applied to US of solid breast nodules by using neural networks, Radiology 213 (1999) 407-412.

[12] S. Joo, Y. S. Yang, W. K. Moon, H. C. Kim, Computeraided diagnosis of solid breast nodules: use of an artificial neural network based on multiple sonographic features, IEEE Transactions on Medical Imaging 23 (2004) 1292-1300.

[13] C.-K. Yeh, Y.-S. Chen, W.-Z. Fan, W.-S. Chen, A novel method for automatic contour extraction of ultrasonic breast lesions, in: IEEE Ultrasonics Symposium, 2005, volume 2, IEEE, pp. 13841387.

[14] D. Boukerroui, A. Baskurt, J. A. Noble, O. Basset, Segmentation of ultrasound images - multiresolution 2D and 3D algorithm based on global and local statistics, Pattern Recognition Letters 24 (2003) 779-790.

[15] G. Xiao, M. Brady, J. A. Noble, Y. Zhang, Segmentation of ultrasound B-mode images with intensity inhomogeneity correction, IEEE Transactions on Medical Imaging 21 (2002) 48-57.

[16] A. K. Jumaat, W. E. Z. W. Rahman, A. Ibrahim, R. Mahmud, Segmentation and characterization of masses in breast ultrasound images using active contour, in: 2011 IEEE International Conference on Signal and Image Processing Applications (ICSIPA), IEEE, pp. 404-409.

[17] Y.-L. Huang, Y.-R. Jiang, D.-R. Chen, W. K. Moon, Level set contouring for breast tumor in sonography, Journal of Digital Imaging 20 (2007) 238-247.

[18] R.-F. Chang, W.-J. Wu, W. K. Moon, D.-R. Chen, Automatic ultrasound segmentation and morphology based diagnosis of solid breast tumors, Breast Cancer Research and Treatment 89 (2005) 179-185.

[19] W.-L. Chang, C.-C. Chang, W.-M. Chen, Computer-aided diagnosis system for variance estimation of $3 \mathrm{~d}$ ultrasonography based on gabor filter, in: 2010 International Conference on Biomedical Engineering and Computer Science, IEEE, pp. 14.

[20] A.-I. Sarpe, Image segmentation with clustering K-means and watershed transform, in: 2010 Second International Conferences on Advances in Multimedia, IEEE, pp. 13-17.

[21] D. Boukerroui, O. Basset, N. Guerin, A. Baskurt, Multiresolution texture based adaptive clustering algorithm for breast lesion segmentation, European Journal of Ultrasound 8 (1998) 135144.

[22] D.-R. Chen, R.-F. Chang, W.-J. Kuo, M.-C. Chen, Y.-L. Huang, Diagnosis of breast tumors with sonographic texture analysis using wavelet transform and neural networks, Ultrasound in Medicine \& Biology 28 (2002) 1301-1310.

[23] Y.-L. Huang, D.-R. Chen, Watershed segmentation for breast tumor in 2-D sonography, Ultrasound in Medicine \& Biology 30 (2004) 625-632.

[24] K. Drukker, M. L. Giger, K. Horsch, M. A. Kupinski, C. J. Vyborny, E. B. Mendelson, Computerized lesion detection on breast ultrasound, Medical Physics 29 (2002) 1438-1446.

[25] J. Shan, H. Cheng, Y. Wang, Completely automated segmentation approach for breast ultrasound images using multiple-domain features, Ultrasound in Medicine \& Biology 38 (2012) 262-275.

[26] Y. Su, Y. Wang, J. Jiao, Y. Guo, Automatic detection and classification of breast tumors in ultrasonic images using texture and morphological features., Open Med Inform J 5 (2011) 2637.

[27] B. Liu, H.-D. Cheng, J. Huang, J. Tian, X. Tang, J. Liu, Fully automatic and segmentation-robust classification of breast tumors based on local texture analysis of ultrasound images, Pattern Recognition 43 (2010) 280-298.
[28] A. J. Smola, B. Schölkopf, A tutorial on Support Vector Regression, Statistics and Computing 14 (2004) 199-222.

[29] G. J. McLachlan, Discriminant analysis and statistical pattern recognition, volume 544, John Wiley \& Sons, 2004.

[30] P. Perona, J. Malik, Scale-space and edge detection using anisotropic diffusion, IEEE Transactions on Pattern Analysis and Machine Intelligence 12 (1990) 629-639.

[31] J.-J. Shyu, S.-C. Pei, Y.-D. Huang, Design of variable twodimensional FIR digital filters by McClellan transformation, IEEE Transactions on Circuits and Systems I: Regular Papers 56 (2008) 574-582.

[32] E. Izquierdo, M. Ghanbari, Texture smoothing and object segmentation using feature-adaptive weighted Gaussian filtering, in: ITS Proceedings, volume 2, SBT/IEEE International, pp. 650-655.

[33] I. Soares, M. Castelo-Branco, A. M. Pinheiro, Curvature detection and segmentation of retinal exudates, in: 12 9th IEEE International Symposium on Biomedical Imaging (ISBI), IEEE, pp. 1719-1722.

[34] F. Voci, S. Eiho, N. Sugimoto, H. Sekibuchi, Estimating the gradient in the Perona-Malik equation, IEEE Signal Processing Magazine 21 (2004) 39-65.

[35] Y. Freund, R. Schapire, N. Abe, A short introduction to boosting, Journal of Japanese Society for Artificial Intelligence 14 (1999) 1612.

[36] T. F. Chan, L. A. Vese, Active contours without edges, IEEE Transactions on image processing 10 (2001) 266-277. 